Proyecciones

Vol. 22, $\mathrm{N}^{o}$ 1, pp. 31-62, May 2003.

Universidad Católica del Norte

Antofagasta - Chile

DOI: 10.4067/S0716-09172003000100003

\title{
CARACTERISATION DES RELATIONS BINAIRES FINIES D-DEMI-RECONSTRUCTIBLES
}

\author{
JAMEL DAMMAK \\ Université Claude Bernard Lyon 1, France
}

\begin{abstract}
Given a binary relation $R$ of basis $E$, we define its dual $R^{\star}$ by $R^{\star}(x, y)=R(y, x)$. A relation $R$ is self-dual if it is isomorphic to $R^{\star}$. $A$ binary relation $R^{\prime}$ is hemimorphic to $R$, if it is isomorphic to $R$ or to $R^{\star}$. A binary relation $R$ is d-half-reconstructible if it is determined by its restrictions of cardinality $d$, up to hemimorphism. In this paper we characterize the finite binary relations $d$-half-reconstructibile for every $d \in\{7,8,9,10,11\}$.
\end{abstract}

MSC : 03C60; 04A05; 05C20;05C38; $05 C 40$.

Key Words : Relation de différence, Relation binaire, Graphe, Hypomorphe, Hémimorphe, Reconstruction. 


\section{Introduction.}

Soit $E$ un ensemble fini. Le cardinal de $E$ est noté $|E|$. Une relation binaire $R$ de base $E$ est une application du produit $E \times E$ dans $\{+,-\}$. Les éléments de $E$ sont appelés sommets de $R$. Les paires de sommets sont appelées arêtes de $R$. Une arête $\{a, b\}$ est dite neutre si $R(a, b)=R(b, a)$, elle est dite pleine (resp. vide) si $R(a, b)=R(b, a)=+(\operatorname{resp} . R(a, b)=R(b, a)=-)$. Une arête de $R$ est dite orientée, si elle n'est pas neutre, dans ce cas on dit que $a$ domine $b$ si $R(a, b)=+$. On appelle duale de $R$, la relation $R^{\star}$ définie sur $E$ par : pour tout élément $x \in E, R^{\star}(x, x)=R(x, x)$ et pour tous éléments distincts $x, y \in E, R^{\star}(x, y)=R(y, x)$. La relation $R$ est dite auto-duale si elle est isomorphe à $R^{\star}$. L'isomorphie entre deux relations $R$ et $R^{\prime}$ est notée $R \sim R^{\prime}$. Une relation binaire $T$ est un tournoi lorsque pour tous $x, y$ éléments distincts de sa base, $T(x, x)=-$ et $T(x, y) \neq T(y, x)$. Un 3-cycle $(a, b, c)$ est un tournoi $T$ à 3 éléments défini par $T(a, b)=T(b, c)=T(c, a)=+$. Une $k$-chaîne est un ordre total irréflexif à $k$ éléments. Un diamant positif (resp. négatif) est un tournoi à 4 sommets constitué d'un 3-cycle $(a, b, c)$ et d'un point $d$ dominé par (resp. dominant) $(a, b, c)$. Le point $d$ est dit sommet du diamant. La restriction de $R$ à une partie $X$ de $E$, notée $R / X$, est la relation de base $X$ définie par : pour tous éléments $x, y$ de $X, R / X(x, y)=R(x, y)$.

Soient $R$ et $R^{\prime}$ deux relations binaires de bases respectives $E$ et $E^{\prime}$, et $f$ une bijection de $E$ sur $E^{\prime}$. L'application $f$ est dite un isomorphisme (resp. anti-isomorphisme) de $R$ sur $R^{\prime}$, si pour tous $x, y$ éléments de $E$, $R^{\prime}(f(x), f(y))=R(x, y)$ (resp. $\left.R^{\prime}(f(x), f(y))=R^{\star}(x, y)\right)$. Dans ce cas on dit que $R$ et $R^{\prime}$ sont isomorphes et on note $R^{\prime} \sim R$ (resp. $R$ et $R^{\prime}$ sont anti-isomorphes et on note $R^{\prime} \sim R^{\star}$ ). Une relation binaire $R^{\prime}$ est hémimorphe à $R$, si elle est isomorphe à $R$ ou à $R^{\star}$. Une relation binaire $R$ est dite auto-duale si elle est isomorphe à $R^{\star}$. Une relation binaire est dite non auto-duale minimale, si elle est non auto-duale et si toutes ses restrictions strictes sont auto-duales. Une relation binaire $H$ s'abrite dans $R$ si $H$ est isomorphe à une restriction de $R$. Une restriction $R / A$ s'inverse dans $R^{\prime}$ si $R^{\prime} / A \sim R^{\star} / A$. Deux relations binaires $R$ et $R^{\prime}$ de base commune $E$ de cardinal $n$ sont dites $k$-hémimorphes (resp. $k$-hypomorphes) lorsque pour toute partie $X$ de $E$ de cardinal $k, R^{\prime} / X \sim R / X$ ou $R^{\prime} / X \sim R^{\star} / X$ (resp. $R^{\prime} / X \sim R / X$ ); définition analogue lorsque $\operatorname{card}(X) \leq k$, on remplace alors le préfixe $(k)$ par $(\leq k)$ dans les notations. Une relation $R$ est dite $k$-demi-reconstructible (resp. $k$-reconstructible) si toute relation 
$k$-hémimorphe (resp. $k$-hypomorphe) à $R$, lui est hémimorphe (resp. isomorphe); définition analogue pour la $(\leq k)$-demi-reconstructibilité (resp. $(\leq k)$-reconstructibilité). J. G. Hagendorf et G. Lopez ont montré [4] que les relations binaires finies sont $(\leq 12)$-demi-reconstructibles. J. Dammak [1] a amélioré ce résultat comme suit : Pour tout entier $k \geq 2$, les relations binaires finies abritant un non auto-dual de cardinal $k$, sont $(\leq k+6)$ demi-reconstructibles. Ces résultats ont motivé l'étude que nous faisons ici, la caractérisation des relations binaires finies $d$-demi-reconstructibiles pour tout $d \in\{7,8,9,10,11\}$, par exemple pour $d=11$, nous montrons :

Théorème 1.1. Soient $R$ une relation binaire de base finie $E$ de cardinal au moins égal à 21 et $k$ le plus petit cardinal des restrictions non autoduales de $R$. Alors $R$ n'est pas 11-demi-reconstructible si et seulement si $k=6$ et $R$ admet au moins deux composantes connexes non auto-duales qui sont des tournois sans diamant.

Esquisse de la preuve du théorème 1. La démarche est sensiblement la même pour les autres théorèmes de caractérisation. On considère deux relations binaires $R$ et $R^{\prime}, 11$-hémimorphes définies sur une base finie $E$ de cardinal au moins égal à 21 . Soit $k$ le plus petit cardinal des restrictions non auto-duales de $R$ et $I_{0}$ la base de l'une de ces restrictions. D'après [1], les relations binaires connexes sont 7-demi-reconstructibles donc également 11-demi-reconstructibles. On peut supposer alors que $R$ possède au moins deux composantes connexes. D'autre part d'après [1] les relations binaires finies abritant un non auto-dual de cardinal $k$, sont $(\leq k+6)$-demi-reconstructibles. Donc si $2 \leq k \leq 5$, alors $R$ est 11-demireconstructible. Si $k=6$ et si $R / I_{0} \sim R^{\prime} / I_{0}$, alors les relations $R$ et $R^{\prime}$ sont 5-hypomorphes et par suite nous montrons que si $C$ est une classe de l'équivalence $D_{R, R^{\prime}}$ et si $C$ n'est pas un tournoi sans diamant, alors $R^{\prime} / C \sim R / C \sim R^{\star} / C$. Il s'ensuit que si $R$ admet au plus une composante connexe non auto-duale qui est un tournoi sans diamant, alors $R$ est 11demi-reconstructible. Si $R$ admet au moins deux composantes connexes non auto-duales qui sont des tournois sans diamant, alors $R$ n'est pas 11demi-reconstructible. Dans le cas $k \geq 7$ ou $k=0$, la relation $R$ n'admet aucune restriction non auto-duale de cardinal inférieur ou égal à 6 , et par suite $R$ et $R^{\prime}$ sont 6-hypomorphes. Il s'ensuit que $R \sim R^{\prime}$ (Voir [5]). Donc $R$ est encore 11-demi-reconstructible. 


\section{Définitions. Notations. Rappels.}

Somme lexicographique. Etant donnée une relation $S$ de base $I=$ $\{1, \ldots, k\}$, associons à chaque $i \in I$, une relation $R_{i}$ de base $I_{i}$ de telle sorte que les bases $I_{i}$ soient deux à deux disjointes. La $S$-somme des $R_{i}$, notée $S\left(R_{1}, \ldots, R_{k}\right)$, est la relation définie sur la réunion des $I_{i}$ de la façon suivante $: S\left(R_{1}, \ldots, R_{k}\right)(x, y)=R_{i}(x, y)$ si $x, y \in I_{i}$ et $S\left(R_{1}, \ldots, R_{k}\right)(x, y)=S(i, j)$ si $x \in I_{i}$ et $y \in I_{j}$. Nous dirons aussi que la $S$-somme des $R_{i}$, est obtenue à partir de la relation $S$ en dilatant chaque $i \in I$ par la relation $R_{i}$.

Relations particulières. Citons les relations irréflexives particulières suivantes :

- Une presque-chaîne de longueur $k$ est obtenue à partir d'une $k$-chaîne en rendant neutre l'arête liant son premier et son dernier élément. Une presque-chaîne de longueur 3 est dite aussi une 3-consécutivité.

- Un pic est une relation à 3 éléments $a, b$ et $c$ telle que l'arête $\{a, b\}$ est neutre et les arêtes $\{a, c\},\{b, c\}$ sont orientées avec $R(a, c)=R(b, c)$.

- Un drapeau est une relation à 3 éléments $a, b$ et $c$ telle que l'arête $\{a, b\}$ est orientée, l'arête $\{b, c\}$ est pleine et l'arête $\{a, c\}$ est vide.

- Etant donné un entier $h, T_{h}$ est le tournoi à $2 h+1$ sommets : $0,1, \ldots, 2 h$ tel que pour tout sommet $i, T_{h}(i, i+k)=+$ pour $k \in\{1, \ldots, h\}$, (l'entier $i+k$ étant considéré modulo $2 h+1)$. Une relation $R$ est un élément de $D\left(T_{h}\right)$, si $R$ est un tournoi obtenu en dilatant chaque sommet $k$ de $T_{h}$ par une chaîne $p_{k}$ de cardinal fini. Rappelons que $D\left(T_{h}\right)$ est la classe des tournois finis sans diamant $([3],[6])$.

- Soient un entier naturel $h \geq 3$ et l'ensemble $F=\{1, \ldots, h\}$.

* On appelle consécutivité $1<2<\ldots<h$, l'une des quatre relations définies sur $F$ comme suit:

$\left[R_{1}(x, y)=+\operatorname{ssi}(y=x+1)\right],\left[R_{2}(x, y)=+\operatorname{ssi}(y=x+1\right.$ ou $\left.y=x)\right]$, $\left[R_{3}(x, y)=+\operatorname{ssi}(y=x+1\right.$ ou $\left.|y-x|>1)\right],\left[R_{4}(x, y)=+\right.$ ssi $(y=$ $x+1$ ou $|y-x| \neq 1)]$.

* On appelle cycle $1<2<\ldots<h<1$, l'une des quatre relations 
définies sur F comme suit : pour toute consécutivité $R_{i},(1 \leq i \leq 4)$, le cycle $R_{i}^{\prime}$ coïncide avec $R_{i}$, sauf peut être sur les couples $(1, h)$ et $(h, 1)$ où on a $R_{i}^{\prime}(h, 1)=+$ et $R_{i}^{\prime}(1, h)=-$.

- Etant donné un entier $n \geq 1$, on désigne par $S_{n}$, l'une des relations définies sur les $2 n$ éléments $t_{1}, \ldots, t_{2 n}$, par : $S_{n}\left(t_{i}, t_{i+n}\right)=S_{n}\left(t_{i+n}, t_{i}\right)$ pour $i=1, \ldots, n, S_{n}\left(t_{i}, t_{i+k}\right) \neq S_{n}\left(t_{i+k}, t_{i}\right)=+$ pour $k=1, \ldots, n-1$ et pour $i=1, \ldots, 2 n$ (les entiers ici sont considérés modulo $2 n$ ). On notera $\delta_{n}$ un élément de la famille $E\left(S_{n}\right)$, des extensions de $S_{n}$ obtenues en augmentant la base de $S_{n}$ d'ensembles (éventuellement vides) deux à deux disjoints $s_{1}, \ldots, s_{2 n}$, appelés secteurs de la relation, tels que :

i) Pour tout $i, \delta_{n} / s_{i} \cup\left\{t_{i}, t_{i+1}\right\}$ est une chaîne de premier élément $t_{i}$ et de dernier élément $t_{i+1}$.

ii) Pour tout $i$, on a $\delta_{n} / s_{i} \cup s_{i+n}$ est un tournoi sans diamant.

iii) Pour tout $i$, pour tout $x$ de $s_{i}$, pour tout $y$ de $s_{i+j},(j=1, \ldots, n-1)$, on a $\delta_{n}(x, y)=-, \delta_{n}(y, x)=+, \delta_{n}\left(t_{i}, y\right) \neq \delta_{n}\left(y, t_{i}\right)=+$, et pour tout $y$ de $s_{i+j},(j=n, \ldots, 2 n-1)$, on a $\delta_{n}\left(t_{i}, y\right) \neq \delta_{n}\left(y, t_{i}\right)=-$.

Intervalle, Décomposabililité. La notion suivante d'intervalle d'une relation binaire a été introduite par R. Fraïssé en [2]. Etant donnée une relation binaire $R$ de base $E$, une partie $I$ de $E$ est un $R$-intervalle, lorsque pour tous éléments $a, b \in I$, tels que $R(a, a)=R(b, b)$, et pour tout élément $x \in E-I$, on a $R(a, x)=R(b, x)$ et $R(x, a)=R(x, b)$. Clairement, l'ensemble vide, les singletons et l'ensemble $E$ sont des intervalles de $R$, appelés intervalles triviaux. Une relation ayant au moins trois sommets sera dite indécomposable lorsque tous ses intervalles sont triviaux, elle est dit décomposable dans le cas contraire. Si $I$ et $J$ sont deux $R$-intervalles, la valeur $R(a, b)$ est une constante quand $a$ (resp. $b$ ) décrit l'ensemble $I$ (resp. $J$ ) et on note $R(I, J)$ cette constante.

Rappelons les résultats suivants :

Lemme 2.1. [5] Les relations binaires finies sont $(\leq 6)$-reconstructibles.

Lemme 2.2. [4] Les relations binaires finies sont $(\leq 12)$-demi-reconstructibles. 
Dans le cas des relations binaires connexes ce seuil a été abaissé dans [1] :

Lemme 2.3. [1] Les relations binaires connexes finies sont $(\leq 7)$-demireconstructibles.

Lemme 2.4. [1] Pour tout entier $k \geq 2$, les relations binaires finies abritant un non auto-dual de cardinal $k$, sont $(\leq k+6)$-demi-reconstructibles.

Lemme 2.5. [8]. Soient $R$ et $R^{\prime}$ deux relations binaires de même base $E$ de cardinal fini $n$. Si $R$ et $R^{\prime}$ sont q-hémimorphes (resp. q-hypomorphes) où $1 \leq q \leq n-1$, alors pour tout entier $p \leq \min (q, n-q)$, $R$ et $R^{\prime}$ sont p-hémimorphes (resp. p-hypomorphes).

Relation de différence. La notion de relation de différence a été introduite par G. Lopez dans [5]. Soient deux relations binaires $R$ et $R^{\prime}$ de même base $E$, qui sont $(\leq 2)$-hémimorphes. On définit la relation $D_{R, R^{\prime}}$ de base $E$ par : pour tout élément $x$ de $E, D_{R, R^{\prime}}(x, x)=+$ et pour tous éléments distincts $x, y$ de $E, D_{R, R^{\prime}}(x, y)=+$ lorsqu'il existe une suite $x=x_{0}, x_{1}, \ldots, x_{k}=y$ d'éléments de $E$ telle que $R\left(x_{i}, x_{i+1}\right) \neq R^{\prime}\left(x_{i}, x_{i+1}\right)$ pour tout $i$ élément de $\{0,1, \ldots, k-1\}$. La relation $D_{R, R^{\prime}}$ est une relation d'équivalence appelée relation de différence dont les classes sont appelées classes de différence.

Rappelons les résultats suivants :

Lemme 2.6. [6] Soient $R$ et $R^{\prime}$ deux relations binaires sur une même base finie $E$, $(\leq 3)$-hypomorphes, et soit $C$ une classe de l'équivalence $D_{R, R^{\prime}}$, alors : $R^{\prime}$.

i) Toute classe de l'équivalence $D_{R, R^{\prime}}$ est un intervalle commun à $R$ et

ii) Les restrictions de $R$ et $R^{\prime}$ à une classe de l'équivalence $D_{R, R^{\prime}}$ sont toutes deux, réflexives ou irréflexives.

iii) Si $C_{1}$ et $C_{2}$ sont deux classes différentes de l'équivalence $D_{R, R^{\prime}}$, alors pour tous $(a, b),(c, d) \in C_{1} \times C_{2}$ on a : $R(a, b)=R(c, d)=R^{\prime}(a, b)=$ 
$R^{\prime}(c, d)$ et $R(b, a)=R(d, c)=R^{\prime}(b, a)=R^{\prime}(d, c)$.

iv) Si $R / C$ n'est pas un tournoi, alors $R / C$ admet une 3-consécutivité.

v) Les 3-consécutivités de $R / C$ s'inversent dans $R^{\prime} / C$.

Lemme 2.7. [6] Soient $R$ et $R^{\prime}$ deux relations binaires $(\leq 4)$-hypomorphes sur une base finie $E$, et $C$ une classe de l'équivalence $D_{R, R^{\prime}}$. Alors :

i) Si $R / C$ est un tournoi, alors il existe un entier $h$ tel que $R / C$ est un $D\left(T_{h}\right)$.

ii) Si $R / C$ n'abrite pas de 3-cycle, alors $R / C$ est soit une chaîne, soit une presque-chaîne, soit une consécutivité, soit un cycle.

iii) Si $R / C$ abrite un 3-cycle, et si $R / C$ n'est pas un tournoi, alors il existe un entier $n$ tel que $R / C$ est un élément de $E\left(S_{n}\right)$.

iv) Les 3-cycles de $R / C$ s'inversent dans $R^{\prime} / C$.

Relation connexe. Une relation $\mathrm{R}$ de base finie $E$ est dite connexe, si pour tous éléments distincts $x, y$ de $E$, il existe un chemin orienté reliant $x$ à $y$, (c'est à dire une suite $x_{0}=x, x_{1}, \ldots, x_{n}=y$, telle que pour tout $i=0,1, \ldots, n-1$, on a $\left.R\left(x_{i}, x_{i+1}\right) \neq R\left(x_{i+1}, x_{i}\right)\right)$. La composante connexe d'une partie $A$ de $E$, telle que $R / A$ est connexe, est la plus grande partie $D$ de $E$ telle que $D$ contient $A$ et la restriction $R / D$ soit connexe.

Relation fortement connexe. Une relation $\mathrm{R}$ de base finie $E$ est dite fortement connexe, si pour tous éléments distincts $x, y$ de $E$, il existe un chemin monotone orienté reliant $x$ à $y$, c'est à dire une suite $x_{0}=$ $x, x_{1}, \ldots, x_{n}=y$, telle que pour tout $i=0,1, \ldots, n-1$, on a $R\left(x_{i}, x_{i+1}\right) \neq$ $R\left(x_{i+1}, x_{i}\right)$ et $R\left(x_{i}, x_{i+1}\right)=+$. La composante fortement connexe de $R$ contenant $x$ est la plus grande partie $D(x)$ de $E$ telle que $D(x)$ contienne $x$ et la restriction $R / D(x)$ soit fortement connexe.

Relations binaires non auto-duales minimales. Une relation binaire est dite non auto-duale minimale si elle est non auto-duale et toutes ses restrictions propres sont auto-duales. 
Rappelons les résultats suivants :

Lemme 2.8. [1] Soient $r \geq 2$ un entier, $R$ et $R^{\prime}$ deux relations binaires $(\leq 4)$-hémimorphes sur une base finie $E$. Si $R$ n'abrite aucune restriction non auto-duale à au plus $r$ sommets, alors les relations $R, R^{\star}$ et $R^{\prime}$ sont deux à deux $(\leq r)$-hypomorphes.

Lemme 2.9. [1] Soient $R$ et $R^{\prime}$ deux relations binaires $(\leq 5)$-hémimorphes sur une base finie $E$, et $C$ une classe de l'équivalence $D_{R, R^{\prime}}$, admettant une restriction non auto-duale, $k$ la plus petite cardinalité des restrictions non auto-duales de $R / C$ et $I_{0}$ la base d'une de ces restrictions. Alors la restriction $R^{\prime} / I_{0}$ est isomorphe à $R^{\star} / I_{0}$.

Notons que comme conséquence des lemmes 2.8 et 2.9 , on a :

Corollaire 2.10. Soit $r$ un entier, $2 \leq r \leq 6$. Soient $R$ et $R^{\prime}$ deux relations binaires $(\leq 5)$-hémimorphes sur une base finie $E, C$ une classe de l'équivalence $D_{R, R^{\prime}}$. Alors $R / C$ et $R^{\prime} / C$ sont $(\leq r)$-hypomorphes ssi $R / C$ n'abrite aucune relation non auto-duale minimale à au plus $r$ sommets.

Lemme 2.11. [1] Soient $d$ un entier au moins égal à $4, R$ et $R^{\prime}$ deux relations binaires $(\leq d)$-hémimorphes sur une base finie $E$ admettant une restriction non auto-duale, $k$ la plus petite cardinalité des restrictions non auto-duales minimales de $R, I_{0}$ la base d'une de ces restrictions et $C$ une classe de l'équivalence $D_{R, R^{\prime}}$, alors :

i) Si $C$ est différente de sa composante connexe, alors $C$ est un intervalle de $R$ et $R^{\prime}$, sur lequel les restrictions de $R$ et $R^{\prime}$ sont réflexives ou irréflexives.

ii) Si $d \geq 5,\left|I_{0}\right|=2$, et si $R / I_{0} \sim R^{\prime} / I_{0}$, alors $C$ est un intervalle de $R$ et $R^{\prime}$, sur lequel les restrictions de $R$ et $R^{\prime}$ sont réflexives ou irréflexives.

iii) Si $d \geq 6$, si $R / I_{0}$ est un drapeau, et si $R / I_{0} \sim R^{\prime} / I_{0}$, alors $C$ est un intervalle de $R$ et $R^{\prime}$, sur lequel les restrictions de $R$ et $R^{\prime}$ sont réflexives ou irréflexives.

Lemme 2.12. [1] Soient $d$ un entier, $d \geq 6$, $R$ et $R^{\prime}$ deux relations binaires $(\leq d)$-hémimorphes sur une base finie $E$, admettant une restriction non auto-duale, $C$ une classe de l'équivalence $D_{R, R^{\prime}}, k$ la cardinalité minimale des restrictions non auto-duales qui s'abritent dans $R$, et $I_{0}$ la base 
d'une de ces restrictions. Si $R / I_{0} \sim R^{\prime} / I_{0}$, alors :

i) Si $C$ est égale à sa composante connexe et si $R / I_{0}$ n'est pas un drapeau, alors $I_{0} \cap C=\phi$.

ii) Si $C$ est égale à sa composante connexe et si $R / I_{0}$ est un drapeau, d'arête neutre $\{c, a\}$ et $\{c, b\}$, alors $I_{0} \cap C=\phi$ ou $I_{0} \cap C=\{c\}$.

Lemme 2.13. [1] Soient $d$ un entier $\geq 5, R$ et $R^{\prime}$ deux relations binaires $(\leq d)$-hémimorphes sur une base finie $E$ admettant une restriction non auto-duale, $k$ la cardinalité minimale des restrictions non auto-duales qui s'abritent dans $R, I_{0}$ la base d'une de ces restrictions et $C$ une classe de l'équivalence $D_{R, R^{\prime}}$.

i) Si $C$ est différente de sa composante connexe, alors $R / C$ et $R^{\prime} / C$ sont $(\leq d-1)$-hypomorphes.

ii) Si $d \geq 6, R^{\prime} / I_{0} \sim R / I_{0}, R / I_{0}$ un drapeau et $C \cap I_{0} \neq \phi$, alors $R / C$ et $R^{\prime} / C$ sont $(\leq d-2)$-hypomorphes.

iii) Si $d \geq 6$ et $R / I_{0} \sim R^{\prime} / I_{0}$, alors $R / C$ et $R^{\prime} / C$ sont $(\leq \max (k-1, d-$ $k)$ )-hypomorphes.

\section{Caractérisation des relations binaires 11-demi-reconstructi- bles}

Dans ce paragraphe nous obtenons :

Théorème 3.1. Soient $R$ une relation binaire de base finie $E$ de cardinal au moins égal à 21 et $k$ le plus petit cardinal des restrictions non autoduales de $R$. Alors $R$ n'est pas 11-demi-reconstructible si et seulement si $k=6$ et $R$ admet au moins deux composantes connexes non auto-duales qui sont des tournois sans diamant.

La preuve du théorème 3.1 se base sur les résultats suivants :

Lemme 3.2. Soient $R$ et $R^{\prime}$ deux relations binaires $(\leq 5)$-hémimorphes sur une base finie $E$ et $C$ une classe de l'équivalence $D_{R, R^{\prime}}$. 
i) Si $R / C$ et $R^{\prime} / C$ sont $(\leq 4)$-hypomorphes et si $R / C$ n'est ni un tournoi sans diamant ni un élément de $E\left(S_{n}\right)$, alors $R^{\prime} / C \sim R / C \sim R^{\star} / C$.

ii) Si $R / C$ et $R^{\prime} / C$ sont $(\leq 5)$-hypomorphes et si $R / C$ est un élément de $E\left(S_{n}\right)$, alors $R^{\prime} / C \sim R / C \sim R^{\star} / C$.

Preuve. i) Le lemme 2.7 de G. Lopez et C. Rauzy nous donne la forme de $R / C$ et $R^{\prime} / C$. D'après la forme si $R / C$ n'est ni un tournoi sans diamant ni un élément de $E\left(S_{n}\right)$ alors $R^{\prime} / C \sim R / C \sim R^{\star} / C$.

ii) Soit $R / C$ un élément de $E\left(S_{n}\right)$, puisque $R / C$ et $R^{\prime} / C$ sont $(\leq 5)$ hypomorphes, alors d'après le lemme $2.6 R / C$ est réflexive ou irréflexive. Sans perte de généralité, on peut supposer que $R / C$ est irréflexive. Comme $R / C$ et $R^{\prime} / C$ sont $(\leq 5)$-hypomorphes, le corollaire 2.10 prouve que $R / C$ n'abrite aucune restriction non auto-duale de cardinal $\leq 5$, et par suite $n \leq 3$. En effet, si $n \geq 4$ et si $\left\{t_{1}, t_{1+n}\right\},\left\{t_{2}, t_{2+n}\right\},\left\{t_{3}, t_{3+n}\right\}$ et $\left\{t_{4}, t_{4+n}\right\}$ sont 4 arêtes neutres de $R / C$, alors la restriction $R /\left\{t_{1}, t_{1+n}, t_{2}, t_{3}, t_{4+n}\right\}$ est une restriction non auto-duale de cardinal $\leq 5$. Suivant la valeur de l'entier $n$, on a :

a) Si $n=3$, alors $R / C=S_{3}$. En effet soient $\left\{t_{1}, t_{4}\right\},\left\{t_{2}, t_{5}\right\}$ et $\left\{t_{3}, t_{6}\right\}$ les 3 arêtes neutres de $R / C$ et supposons que le secteur $s_{1}$ contienne un élément $x$, alors $R /\left\{t_{1}, t_{4}, x, t_{2}, t_{6}\right\}$ est une restriction non auto-duale de cardinal 5. Donc $R / C=S_{3}$, et les arêtes neutres sont toutes de même type car $R / C$ n'abrite aucune restriction non auto-duale de cardinal $\leq 5$.

b) Si $n=2$. Comme $R / C$ n'abrite aucune restriction non auto-duale de cardinal $\leq 5$, alors $R / C$ est, soit un $S_{2}$, soit un élément de $E\left(S_{2}\right)$ avec 3 secteurs vides, un secteur de cardinal 1, et deux arêtes neutres de même type.

c) Si $n=1$. On prouve de même que $R / C$ est soit une presque-chaîne, soit un élément de $E\left(S_{1}\right)$ à 5 sommets de secteurs $s_{1}=\{a\}, s_{2}=\{b, c\}$ et tel que $R /\{a, b, c\}$ est un 3-cycle, soit un élément de $E\left(S_{1}\right)$ à 4 sommets.

Dans tous ces cas, on peut voir que $R^{\prime} / C \sim R / C \sim R^{\star} / C$.

Lemme 3.3. Soient $R$ et $R^{\prime}$ deux relations binaires $(\leq 7)$-hémimorphes sur une base finie $E, D$ une composante connexe de $R$ et $k$ le plus petit 
cardinal des restrictions non auto-duales de $R$.

i) Si $k \in\{5,6\}$ et si $R / D$ n'est ni un tournoi sans diamant ni un élément de $E\left(S_{n}\right)$, alors $R^{\prime} / D \sim R / D \sim R^{\star} / D$.

ii) Si $k=6$ et si $R / D$ est un élément de $E\left(S_{n}\right)$, alors $R^{\prime} / D \sim R / D \sim$ $R^{\star} / D$.

Preuve. Soit $D$ une composante connexe de $R$.

i) Comme $R / D$ n'abrite aucune restriction non auto-duale de cardinal inférieur ou égal à 4 , alors $R / D$ et $R^{\star} / D$ sont $(\leq 4)$-hypomorphes. D'autre part $D$ est une classe de l'équivalence $D_{R, R^{\star}}$, alors le lemme 2.7 prouve que si $R / D$ n'est ni un tournoi sans diamant ni un élément de $E\left(S_{n}\right)$, alors $R / D \sim R^{\star} / D$. Comme $R / D$ est connexe, alors le lemme 2.3 prouve que $R^{\prime} / D \sim R / D$ ou $R^{\prime} / D \sim R^{\star} / D$, et par suite $R^{\prime} / D \sim R / D \sim R^{\star} / D$.

ii) Comme $R / D$ n'abrite aucune restriction non auto-duale de cardinal inférieur ou égal à 5 , alors $R / D$ et $R^{\star} / D$ sont $(\leq 5)$-hypomorphes. D'autre part $D$ est une classe de l'équivalence $D_{R, R^{\star}}$, alors le lemme 3.2 prouve que si $R / D$ est élément de $E\left(S_{n}\right)$, alors $R / D \sim R^{\star} / D$. Comme $R / D$ est connexe, alors le lemme 2.3 prouve que $R^{\prime} / D \sim R / D$ ou $R^{\prime} / D \sim R^{\star} / D$, et par suite $R^{\prime} / D \sim R / D \sim R^{\star} / D$.

Lemme 3.4. Soient $d \in\{7,8,9,10,11\}$, $R$ et $R^{\prime}$ deux relations binaires $d$-hémimorphes sur une base finie $E$ à au moins $2 d-1$ éléments, et $k$ le plus petit cardinal des restrictions non auto-duales de $R$. Si $k=6$ et si $R$ admet au plus une composante connexe non auto-duale qui est un tournoi sans diamant, alors $R^{\prime} \sim R$ ou $R^{\prime} \sim R^{\star}$.

Preuve. Le lemme 2.5 prouve que les relations $R^{\prime}$ et $R$ sont $(\leq d)$ hémimorphes. Soit $D$ une composante connexe de $R$. Comme $k=6$, le lemme 3.3 prouve que si $R / D$ n'est pas un tournoi sans diamant alors $R^{\prime} / D \sim R / D \sim R / D^{\star}$. D'autre part $D$ est un intervalle de $R$ sur lequel $R$ est réflexive ou irréflexive. Il s'ensuit que si $R$ n'admet aucune composante connexe non auto-duale qui est un tournoi sans diamant, alors $R^{\prime} \sim R \sim R^{\star}$. Si parmi les composantes connexes de $R$, une seule notée $D_{1}$ est un tournoi sans diamant non auto-dual, alors le lemme 2.3 prouve que $R^{\prime} / D_{1} \sim R / D_{1}$ ou $R^{\prime} / D_{1} \sim R^{\star} / D_{1}$, et par suite $R^{\prime} \sim R$ si $R^{\prime} / D_{1} \sim R / D_{1}$ et $R^{\prime} \sim R^{\star}$ si $R^{\prime} / D_{1} \sim R^{\star} / D_{1}$. 
Lemme 3.5. Soient $d \in\{9,10,11\}, R$ une relation binaire sur une base finie $E$ et $k$ le plus petit cardinal des restrictions non auto-duales de $R$. Si $d-5 \leq k \leq 6$ et si $R$ admet au moins deux composantes connexes $D_{1}$ et $D_{2}$ non auto-duales qui sont des tournois sans diamant, alors $R$ n'est pas $d$-demi-reconstructible.

Preuve. On va distinguer les cas suivants :

Cas 1. $R / D_{2} \sim R^{\star} / D_{1}$. Soit $n_{1}$ (resp. $n_{2}$ ) le nombre de composantes connexes $D$ de $R$ telles que $R / D \sim R / D_{1}$ (resp. $R / D \sim R / D_{1}^{\star}$ ).

- Si $n_{1} \leq n_{2}$, on définit une relation $R^{\prime}$ de base $E$ coïncidant avec $R$ sauf sur $D_{1} \times D_{1}$ où elle coïncide avec $R^{\star}$. Alors on peut voir que les relations $R$ et $R^{\prime}$ sont $(\leq d)$-hémimorphes sans être hémimorphes.

- Si $n_{2}<n_{1}$, on définit une relation $R^{\prime}$ de base $E$ coïncidant avec $R$ sauf sur $D_{2} \times D_{2}$ où elle coïncide avec $R^{\star}$. Alors on peut voir que les relations $R$ et $R^{\prime}$ sont $(\leq d)$-hémimorphes sans être hémimorphes.

Cas 2. $R / D_{2} \sim R / D_{1}$. D'après le cas 1 , on peut supposer que pour toute composante connexe $D$ de $R, R / D$ n'est pas isomorphe à $R^{\star} / D_{1}$. On définit une relation $R^{\prime}$ de base $E$ coincidant avec $R$ sauf sur $D_{1} \times D_{1}$ où elle cö̈ncide avec $R^{\star}$. Alors On peut voir que les relations $R$ et $R^{\prime}$ sont $(\leq d)$-hémimorphes sans être hémimorphes.

Cas 3. $R / D_{1}$ et $R / D_{2}$ ne sont pas hémimorphes. D'après les cas 1 et 2 , on peut supposer que pour toute composante connexe $D$ de $R, R / D$ n'est pas hémimorphe à $R / D_{1}$. On définit une relation $R^{\prime}$ de base $E$ coïncidant avec $R$ sauf sur $D_{1} \times D_{1}$ où elle coïncide avec $R^{\star}$. Alors on peut voir que les relations $R$ et $R^{\prime}$ sont $(\leq d)$-hémimorphes sans être hémimorphes.

Preuve du théorème 3.1. Soient $R$ une relation binaire finie de base $E$ de cardinal au moins égal à 21 et $k$ le plus petit cardinal des restrictions non auto-duales de $R$. On a :

-Si $R$ est connexe, alors le lemme 2.3 prouve que $R$ est 11-demi-reconstructible.

- Si $2 \leq k \leq 5$, alors le lemme 2.4 prouve que $R$ est 11 -demi-reconstructible. 
- Si $k=6$ et si $R$ admet au plus une composante connexe non auto-duale qui est un tournoi sans diamant, alors le lemme 3.4 prouve que $\mathrm{R}$ est 11demi-reconstructible. Si $R$ admet au moins deux composantes connexes non auto-duales qui sont des tournois sans diamant, alors le lemme 3.5 prouve que $R$ n'est pas 11-demi-reconstructible.

- Si $k \geq 7$ ou $k=0$. Dans ce cas $R$ n'admet aucune restriction non auto-duale de cardinal inférieur ou égal à 6 , alors le lemme 2.1 prouve que $R$ est 11-demi-reconstructible.

Exemple de relation 12-demi-reconstructible mais non 11-demireconstructible

On considère :

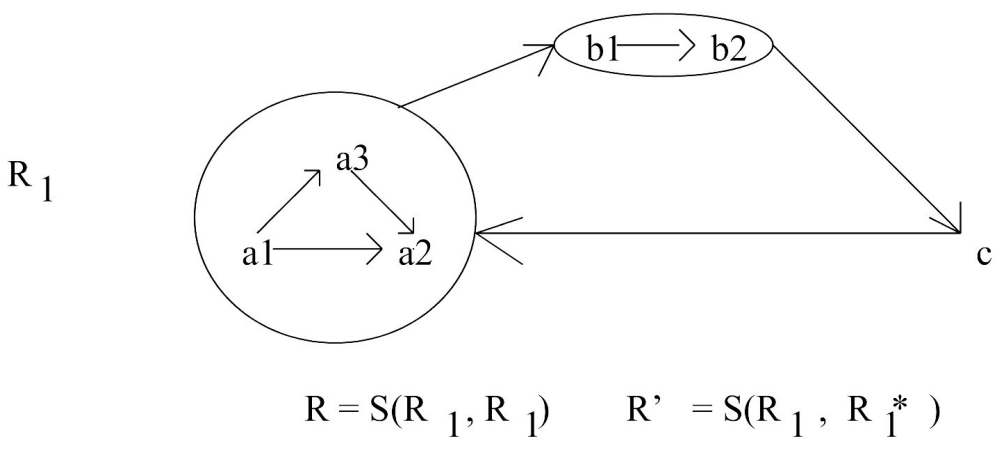

- la relation $R_{1}=C_{3}\left(T_{1}, T_{2}, T_{3}\right)$ où $C_{3}$ est un 3-cycle de base $\{1,2,3\}$ et pour tout $i \in\{1,2,3\}, T_{i}$ est une $i$-chaîne irréflexive.

- $S$ : une relation binaire vide de base $\{1,2\}$.

Sous ces notations, on peut voir que les relations $R=S\left(R_{1}, R_{1}\right)$ et $R^{\prime}=$ $S\left(R_{1}, R^{\star}{ }_{1}\right)$ sont 11-hémimorphes, sans être hémimorphes, et par suite $R$ n'est pas 11-demi-reconstructible. Comme $R_{1}$ est un non auto-dual de cardinal 6 , alors le lemme 2.4 prouve que $R$ est 12-demi-reconstructible.

\section{Caractérisation des relations binaires 10-demi-reconstructi- bles}

Soit $R$ une relation binaire de base $E$. La duale de $R$ à une partie $D$ de $E$, notée $R\left[D^{\star}\right]$, est la relation de base $E$ coïncidant avec $R$ sauf sur $D \times D$ où elle coïncide avec $R^{\star}$. 
Dans ce paragraphe nous obtenons :

Théorème 4.1. Soient $R$ une relation binaire de base finie $E$ de cardinal au moins égal à 19 et $k$ le plus petit cardinal des restrictions non autoduales de $R$. Alors $R$ n'est pas 10-demi-reconstructible si et seulement si elle vérifie l'une des deux conditions suivantes :

- $k \in\{5,6\}$ et $R$ admet au moins deux composantes connexes non autoduales qui sont des tournois sans diamant.

- $k=5$ et parmi les composantes connexes de $R$, une seule notée $D_{1}$ est un tournoi sans diamant non auto-dual et $R^{\star} n$ 'est pas isomorphe à $R\left[D^{\star}{ }_{1}\right]$.

La preuve du théorème 4.1 se base sur les résultats suivants :

Lemme 4.2. Soient $R$ et $R^{\prime}$ deux relations binaires $(\leq 7)$-hémimorphes sur une base finie $E$ et $C$ une classe de l'équivalence $D_{R, R^{\prime}}$. Si $C$ est différente de sa composante connexe alors:

i) $C$ est un intervalle de $R$ et $R^{\prime}$, sur lequel les restrictions de $R$ et $R^{\prime}$ sont réflexives ou irréflexives.

ii) $R^{\prime} / C \sim R / C$.

Preuve. i) Comme $C$ est différente de sa composante connexe, alors le lemme 2.11 prouve que $C$ est un intervalle de $R$ et $R^{\prime}$, sur lequel les restrictions de $R$ et $R^{\prime}$ sont réflexives ou irréflexives.

ii) Comme $C$ est différente de sa composante connexe, alors le lemme 2.13 prouve que $R / C$ et $R^{\prime} / C$ sont $(\leq 6)$-hypomorphes. Il s'ensuit d'après le lemme 2.1 que $R^{\prime} / C \sim R / C$.

Lemme 4.3. Soient $d \in\{7,8,9,10\}, R$ et $R^{\prime}$ deux relations binaires $d$ hémimorphes sur une base finie $E$ de cardinal $n$ au moins égal à $2 d-1, k$ le plus petit cardinal des restrictions non auto-duales de $R$ et $I_{0}$ la base d'une de ces restrictions.

Si $k=d-5$, si aucune composante connexe de $R$ n'est un tournoi sans diamant non auto-dual, et si $R^{\prime} / I_{0} \sim R / I_{0}$, alors $R^{\prime} \sim R$. 
Preuve. Le lemme 2.5 prouve que les relations $R^{\prime}$ et $R$ sont $(\leq d)$ hémimorphes. Soit $C$ une classe de l'équivalence $D_{R, R^{\prime}}$. Comme $R^{\prime} / I_{0} \sim$ $R / I_{0}$, alors d'après le lemme $2.11, C$ est un intervalle de $R$ et $R^{\prime}$ sur lequel $R$ et $R^{\prime}$ sont réflexives ou irréflexives. Si $C$ est différente de sa composante connexe, alors le lemme 4.2 prouve que $R^{\prime} / C \sim R / C$. Dans la suite $C$ est égale à sa composante connexe. Comme $R^{\prime} / I_{0} \sim R / I_{0}$ et $k=d-5$, alors le lemme 2.13 prouve que $R / C$ et $R^{\prime} / C$ sont $(\leq 5)$-hypomorphes. Puisque $R / C$ n'est pas un tournoi sans diamant, alors d'après le lemme 3.2) $R^{\prime} / C \sim R / C$. Il s'ensuit que $R^{\prime} \sim R$.

Lemme 4.4. Soient $d \in\{7,8,9,10\}, R$ et $R^{\prime}$ deux relations binaires $d$ hémimorphes sur une base finie $E$ de cardinal $n$ au moins égal à $2 d-1, k$ le plus petit cardinal des restrictions non auto-duales de $R$ et $I_{0}$ la base d'une de ces restrictions.

Si $k=d-5$, si parmi les composantes connexes de $R$, une seule notée $D_{1}$ est un tournoi sans diamant non auto-dual et si $R^{\prime} / I_{0} \sim R / I_{0}$, alors $R^{\prime} \sim R$ ou $R^{\prime} \sim R\left[D^{\star}{ }_{1}\right]$.

Preuve. Le lemme 2.5 prouve que les relations $R^{\prime}$ et $R$ sont $(\leq d)$ hémimorphes. Comme $R^{\prime} / I_{0} \sim R / I_{0}$, alors d'après le lemme 2.11 toute classe de l'équivalence $D_{R, R^{\prime}}$ est un intervalle de $R$ et $R^{\prime}$ sur lequel $R$ et $R^{\prime}$ sont réflexives ou irréflexives. Le lemme 4.3 prouve que $R^{\prime} /\left(E-D_{1}\right) \sim$ $R /\left(E-D_{1}\right)$, d'autre part $R / D_{1}$ est connexe, alors le lemme 2.3 prouve que $R^{\prime} / D_{1} \sim R / D_{1}$ ou $R^{\prime} / D_{1} \sim R^{\star} / D_{1}$. Il s'ensuit que $R^{\prime} \sim R$ si $R^{\prime} / D_{1} \sim$ $R / D_{1}$ et $R^{\prime} \sim R\left[D^{\star}{ }_{1}\right]$ si $R^{\prime} / D_{1} \sim R^{\star} / D_{1}$.

Preuve du théorème 4.1. Soient $R$ et $R^{\prime}$ deux relations binaires 10hémimorphes sur une base finie $E$ de cardinal au moins égal à 19, $k$ le plus petit cardinal des restrictions non auto-duales de $R$ et $I_{0}$ la base d'une de ces restrictions. Quitte à échanger les rôles de $R$ et $R^{\star}$, on va supposer que $R^{\prime} / I_{0} \sim R / I_{0}$. On a :

$C_{1}$. Si $R$ est connexe, alors le lemme 2.3 prouve que $R$ est 10 -demireconstructible.

$C_{2}$. Si $2 \leq k \leq 4$, alors le lemme 2.4 prouve que $R$ est 10 -demireconstructible. 
$C_{3}$. Si $k=5$, on a les cas suivants :

$C_{31}$. Si $R$ admet au moins deux composantes connexes non auto-duales qui sont des tournois sans diamant, alors le lemme 3.5 prouve que $R$ n'est pas 10-demi-reconstructible.

$C_{32}$. Si aucune composante connexe de $R$ n'est un tournoi sans diamant non auto-dual. Comme $k=d-5(d=10$ et $k=5)$, alors le lemme 4.3 prouve que $R$ est 10-demi-reconstructible.

$C_{33}$. Si parmi les composantes connexes de $R$, une seule notée $D_{1}$ est un tournoi sans diamant non auto-dual et si $R^{\star}$ est isomorphe à $R\left[D^{\star}{ }_{1}\right]$, alors le lemme 4.4 prouve que $\mathrm{R}$ est 10-demi-reconstructible. Si $R^{\star}$ n'est pas isomorphe à $R\left[D^{\star}{ }_{1}\right]$, on peut voir alors que les relations $R$ et $R\left[D^{\star}{ }_{1}\right]$ sont ( $\leq 10)$-hémimorphes sans être hémimorphes. Il s'ensuit que $R$ n'est pas 10-demi-reconstructible.

$C_{4}$. Si $k=6$ et $R$ admet au plus une composante connexe non autoduale qui est un tournoi sans diamant, alors le lemme 3.4 prouve que $\mathrm{R}$ est 10-demi-reconstructible. Si $R$ admet au moins deux composantes connexes non auto-duales qui sont des tournois sans diamant, alors le lemme 3.5 prouve que $R$ n'est pas 10-demi-reconstructible.

$C_{5}$. Si $k \geq 7$ ou $k=0$. Dans ce cas $R$ n'admet aucune restriction non auto-duale de cardinal inférieur ou égal à 6 , alors le lemme 2.1 prouve que $R$ est 10 -demi-reconstructible. 
Exemple de relation 11-demi-reconstructible mais non 10-demireconstructible.

On considère :

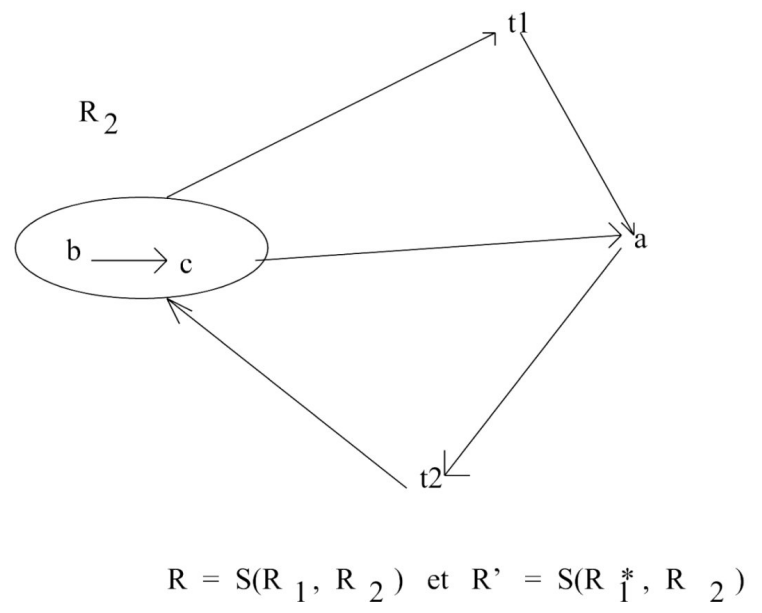

- la relation $R_{1}=C_{3}\left(T_{1}, T_{2}, T_{3}\right)$ où $C_{3}$ est un 3-cycle de base $\{1,2,3\}$ et pour tout $i \in\{1,2,3\}, T_{i}$ est une $i$-chaîne irréflexive.

- la relation $R_{2}$ est une relation irréflexive de base $\left\{t_{1}, t_{2}, a, b, c\right\}$ qui est un élément de $E\left(S_{1}\right)$, d'arête vide $\left\{t_{1}, t_{2}\right\}$, de secteurs $s_{1}=\{a\}$ et $s_{2}=\{b, c\}$ et telle que $R_{2}\left(s_{2}, s_{1}\right)=+$.

- $S$ : une relation binaire vide de base $\{1,2\}$.

Sous ces notations, on peut voir que les relations $R=S\left(R_{1}, R_{2}\right)$ et $R^{\prime}=S\left(R_{1}^{\star}, R_{2}\right)$ sont 10-hémimorphes, sans être hémimorphes, et par suite $R$ n'est pas 10-demi-reconstructible. Comme $R_{2}$ est un non auto-dual de cardinal 5, alors le lemme 2.4 prouve que $R$ est 11-demi-reconstructible.

\section{Caractérisation des relations binaires 9-demi-reconstructibles}

Dans ce paragraphe nous obtenons :

Théorème 5.1. Soient $R$ une relation binaire de base finie $E$ de cardinal au moins égal 17 et $k$ le plus petit cardinal des restrictions non auto-duales 
de $R$. Alors $R$ n'est pas 9-demi-reconstructible si et seulement si $R$ vérifie l'une des conditions suivantes :

- $k=4$ et $R$ admet au moins deux composantes connexes non autoduales qui sont des tournois sans diamant.

- $k=4$ et parmi les composantes connexes de $R$, une seule notée $D_{1}$ est un tournoi sans diamant non auto-dual et $R^{\star}$ n'est pas isomorphe à $R\left[D^{\star}{ }_{1}\right]$.

- $k=5$ et $R$ admet au moins deux composantes connexes non autoduales qui sont des tournois sans diamant ou des éléments de $E\left(S_{n}\right)$.

- $k=6$ et $R$ admet au moins deux composantes connexes non autoduales qui sont des tournois sans diamant.

La preuve du théorème 5.1 utilise les lemmes suivants :

Lemme 5.2. Soient $d \in\{8,9\}, R$ une relation binaire sur une base finie $E$ et $k$ le plus petit cardinal des restrictions non auto-duales de $R$. Si $d-4 \leq k \leq 5$ et $R$ admet au moins deux composantes connexes $D_{1}$ et $D_{2}$ non auto-duales qui sont des tournois sans diamant ou des éléments de $E\left(S_{n}\right)$ alors $R$ n'est pas d-demi-reconstructible.

Preuve. La preuve est analogue à celle du lemme 3.5, car dans la preuve du lemme 3.5 on n'a pas utilisé le fait que $D_{1}$ et $D_{2}$ sont des tournois sans diamant.

Lemme 5.3. Soient $d \in\{7,8,9\}$, $R$ et $R^{\prime}$ deux relations binaires $d$-hémimorphes sur une base finie $E$ à au moins $2 d-1$ éléments, et $k$ le plus petit cardinal des restrictions non auto-duales de $R$.

Si $k=5$ et si $R$ admet au plus une composante connexe $D_{1}$ non auto-duale qui est un tournoi sans diamant ou un élément de $E\left(S_{n}\right)$ alors $R^{\prime} \sim R$ ou $R^{\prime} \sim R^{\star}$.

Preuve. Le lemme 2.5 prouve que les relations $R^{\prime}$ et $R$ sont $(\leq d)$ hémimorphes. Soit $D$ une composante connexe de $R$. Comme $k=5$, alors $R$ et $R^{\star}$ sont $(\leq 4)$-hypomorphes. D'autre part $D$ est une classe de l'équivalence $D_{R, R^{\star}}$, et d'après le lemme 2.6 $\mathrm{D}$ est un intervalle de $R$ et $R^{\prime}$ sur lequel $R$ et $R^{\prime}$ sont réflexives ou irréflexives. Comme $R / D$ est connexe, alors le lemme 2.3 prouve que $R^{\prime} / D \sim R / D$ ou $R^{\prime} / D \sim R^{\star} / D$, 
et par suite si $R / D$ est auto-dual alors $R^{\prime} / D \sim R / D \sim R / D^{\star}$. Comme $k=5$, le lemme 3.3 prouve que si $R / D$ n'est ni un tournoi sans diamant ni un élément de $E\left(S_{n}\right)$ alors $R^{\prime} / D \sim R / D \sim R / D^{\star}$. Il s'ensuit que si $R$ n'admet aucune composante connexe non auto-duale qui est un tournoi sans diamant ou un élément de $E\left(S_{n}\right)$ alors $R^{\prime} \sim R \sim R^{\star}$. De même si parmi les composantes connexes de $R$, une seule non auto-duale notée $D_{1}$ est un tournoi sans diamant ou un élément de $E\left(S_{n}\right)$ alors $R^{\prime} \sim R$ si $R^{\prime} / D_{1} \sim R / D_{1}$ et $R^{\prime} \sim R^{\star}$ si $R^{\prime} / D_{1} \sim R^{\star} / D_{1}$.

Preuve du théorème 5.1. Soient $R$ et $R^{\prime}$ deux relations binaires 9hémimorphes sur une base finie $E$ de cardinal au moins égal à $17, k$ le plus petit cardinal des restrictions non auto-duales de $R$ et $I_{0}$ la base d'une de ces restrictions. Quitte à échanger les rôles de $R$ et $R^{\star}$, on va supposer que $R^{\prime} / I_{0} \sim R / I_{0}$. On a les cas suivants :

$C_{1}$. Si $R$ est connexe, alors le lemme 2.3 prouve que $R$ est 9 -demireconstructible.

$C_{2}$. Si $2 \leq k \leq 3$, alors le lemme 2.4 prouve que $R$ est 9 -demireconstructible.

$C_{3}$. Si $k=4$, on a :

$C_{31}$. Si $R$ admet au moins deux composantes connexes non auto-duales qui sont des tournois sans diamant, alors le lemme 3.5 prouve que $R$ n'est pas 9-demi-reconstructible.

$C_{32}$. Si aucune composante connexe de $R$ n'est un tournoi sans diamant non auto-dual, alors comme $k=d-5 \quad(d=9 \quad$ et $k=4)$, le lemme 4.3 prouve que $R$ est 9-demi-reconstructible.

$C_{33}$. Si parmi les composantes connexes de $R$, une seule notée $D_{1}$ est un tournoi sans diamant non auto-dual, si $R^{\star}$ est isomorphe à $R\left[D^{\star}{ }_{1}\right]$, alors le lemme 4.4 prouve que $R$ est 9 -demi-reconstructible. Si $R^{\star}$ n'est pas isomorphe à $R\left[D^{\star}{ }_{1}\right]$, alors on peut voir que les relations $R$ et $R\left[D^{\star}{ }_{1}\right]$ sont ( $\leq 9)$-hémimorphes sans être hémimorphes. Il s'ensuit que $R$ n'est pas 9demi-reconstructible. 
$C_{4}$. Si $k=5$ et $R$ admet au moins deux composantes connexes non auto-duales qui sont des tournois sans diamant ou des éléments de $E\left(S_{n}\right)$, alors le lemme 5.2 prouve que $R$ n'est pas 9-demi-reconstructible. Si $R$ admet au plus une composante connexe non auto-duale qui est un tournoi sans diamant ou un élément de $E\left(S_{n}\right)$ alors le lemme 5.3 prouve que $R$ est 9-demi-reconstructible.

$C_{5}$. Si $k=6$ et $R$ admet au plus une composante connexe non autoduale qui est un tournoi sans diamant, alors le lemme 3.4 prouve que $R$ est 9-demi-reconstructible. Si $R$ admet au moins deux composantes connexes non auto-duales qui sont des tournois sans diamant alors le lemme 3.5 prouve que $R$ n'est pas 9-demi-reconstructible.

$C_{6}$. Si $k \geq 7$ ou $k=0$. Dans ce cas $R$ n'admet aucune restriction non auto-duale de cardinal inférieur ou égal à 6 , alors le lemme 2.1 prouve que $R$ est 9-demi-reconstructible.

Exemple de relation 10-demi-reconstructible mais non 9-demireconstructible.

On considère :

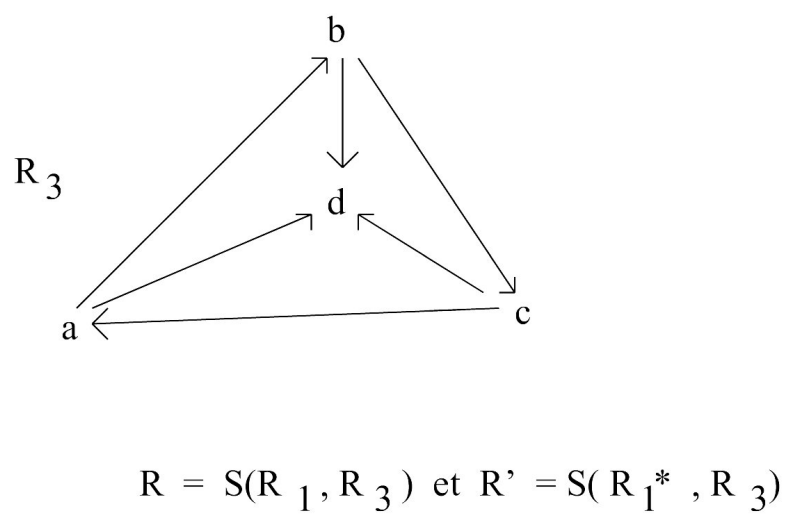

- La relation $R_{1}=C_{3}\left(T_{1}, T_{2}, T_{3}\right)$ où $C_{3}$ est un 3-cycle de base $\{1,2,3\}$ et pour tout $i \in\{1,2,3\}, T_{i}$ est une $i$-chaîne irréflexive.

- La relation $R_{3}$ est un tournoi de base $\{a, b, c, d\}$ prenant la valeur + sur les seuls couples $(a, b),(b, c),(c, a)$ et $(x, d)$ avec $x=a, b, c, R_{3}$ est un diamant. 
- $S$ : une relation binaire vide de base $\{1,2\}$.

Sous ces notations, on peut voir que les relations $R=S\left(R_{1}, R_{3}\right)$ et $R^{\prime}=$ $S\left(R_{1}^{\star}, R_{3}\right)$ sont 9-hémimorphes sans être hémimorphes, et par suite $R$ n'est pas 9-demi-reconstructible. Comme $R_{3}$ est un non auto-dual de cardinal 4 , alors le lemme 2.4 prouve que $R$ est 10-demi-reconstructible.

\section{Caractérisation des relations binaires 8-demi-reconstructibles}

Dans ce paragraphe nous obtenons :

Théorème 6.1. Soient $R$ une relation binaire de base finie $E$ de cardinal au moins égal à 15 et $k$ le plus petit cardinal des restrictions non autoduales de $R$. Alors $R$ n'est pas 8-demi-reconstructible si et seulement si $R$ vérifie l'une des conditions suivantes :

- $k=3$ et $R$ admet au moins deux composantes connexes non autoduales qui sont des tournois sans diamant, et aucun point de ces composantes connexes n'est le sommet d'un drapeau.

- $k=3$ et parmi les composantes connexes de $R$, une seule notée $D_{1}$ est un tournoi sans diamant non auto-dual, disjoint de tout drapeau, et $R^{\star}$ n'est pas isomorphe à $R\left[D^{\star}{ }_{1}\right]$.

- $k=4$ et $R$ admet au moins deux composantes connexes non autoduales qui sont des tournois sans diamant ou des éléments de $E\left(S_{n}\right)$.

- $k=4$, parmi les composantes connexes non auto-duales de $R$, une seule notée $D_{1}$ est un tournoi sans diamant ou un élément de $E\left(S_{n}\right)$, et $R^{\star}$ n'est pas isomorphe à $R\left[D^{\star}{ }_{1}\right]$.

- $k=5$ et $R$ admet au moins deux composantes connexes non autoduales qui sont des tournois sans diamant ou des éléments de $E\left(S_{n}\right)$.

- $k=6$ et $R$ admet au moins deux composantes connexes non autoduales qui sont des tournois sans diamant. 
La preuve du théorème 6.1 se base sur les résultats suivants :

Lemme 6.2. Soient $R$ et $R^{\prime}$ deux relations binaires 8-hémimorphes sur une base finie $E$ de cardinal au moins égal à $15, k$ le plus petit cardinal des restrictions non auto-duales de $R, I_{0}$ la base d'une de ces restrictions et $D$ une composante connexe de $R$. Si $R / I_{0}$ est un drapeau, $D \cap I_{0} \neq \phi$ et $R^{\prime} / I_{0} \sim R / I_{0}$ alors $R^{\prime} / D \sim R / D$.

Preuve. Le lemme 2.5 prouve que les relations $R^{\prime}$ et $R$ sont $(\leq 8)$ hémimorphes. Comme $R^{\prime} / I_{0} \sim R / I_{0}$, alors le lemme 2.11 prouve que toute classe de l'équivalence $D_{R, R^{\prime}}$ est un intervalle de $R$ et $R^{\prime}$, sur lequel $R$ et $R^{\prime}$ sont réflexives ou irréflexives. Si l'équivalence $D_{R / D, R^{\prime} / D}$ possède au moins deux classes, alors d'après le lemme $4.2 R^{\prime} / D \sim R / D$. Dans la suite $D$ est une classe de l'équivalence $D_{R, R^{\prime}}$. Comme $R / I_{0}$ est un drapeau, $R^{\prime} / I_{0} \sim R / I_{0}$ et $D \cap I_{0} \neq \phi$, alors le lemme 2.13 prouve que $R / D$ et $R^{\prime} / D$ sont $(\leq 6)$-hypomorphes. Il s'ensuit, d'après le lemme 2.1, que $R^{\prime} / D \sim R / D$.

Lemme 6.3. Soient $R$ et $R^{\prime}$ deux relations binaires 8-hémimorphes sur une base finie $E$ de cardinal au moins égal $15, k$ le plus petit cardinal des restrictions non auto-duales de $R, I_{0}$ la base d'une de ces restrictions et $D$ une composante connexe de $R$.

Si $k=3$ et si chaque composante connexe de $R$ qui est un tournoi sans diamant est auto-duale ou contient un sommet d'un drapeau, alors $R^{\prime} \sim R$ ou $R^{\prime} \sim R^{\star}$.

Preuve. La preuve est analogue à celle du lemme 4.3.

Lemme 6.4. Soient $R$ et $R^{\prime}$ deux relations binaires 8-hémimorphes sur une base finie $E$ de cardinal au moins égal $15, k$ le plus petit cardinal des restrictions non auto-duales de $R, I_{0}$ la base d'une de ces restrictions et $D$ une composante connexe de $R$.

Si $k=3$, si parmi les composantes connexes de $R$, une seule notée $D_{1}$ est un tournoi sans diamant non auto-dual disjoint de tout drapeau et si $R^{\prime} / I_{0} \sim R / I_{0}$, alors $R^{\prime} \sim R$ ou $R^{\prime} \sim R\left[D^{\star}{ }_{1}\right]$.

Preuve. La preuve est analogue à celle du lemme 4.4. 
Lemme 6.5. Soient $R$ une relation binaire sur une base finie $E$ et $k$ le plus petit cardinal des restrictions non auto-duales de $R$. Si $k=3$ et si $R$ admet au moins deux composantes connexes $D_{1}$ et $D_{2}$ non auto-duales qui sont des tournois sans diamants, et disjoints de tout drapeau, alors $R$ n'est pas 8-demi-reconstructible.

Preuve. La preuve est analogue à la preuve du lemme 3.5.

Lemme 6.6. Soient $R$ et $R^{\prime}$ deux relations binaires 8-hémimorphes sur une base finie $E$ de cardinal au moins égal $15, k$ le plus petit cardinal des restrictions non autohémimorphes-duales de $R$, et $I_{0}$ la base d'une de ces restrictions.

Si $k=4$, et aucune composante connexe non auto-duale de $R$ n'est un tournoi sans diamants ou un élément de $E\left(S_{n}\right)$, et si $R^{\prime} / I_{0} \sim R / I_{0}$, alors $R^{\prime} \sim R$.

Preuve. Le lemme 2.5 prouve que les relations $R^{\prime}$ et $R$ sont $(\leq 8)$ hémimorphes. Soit $C$ une classe de l'équivalence $D_{R, R^{\prime}}$. Comme $R^{\prime} / I_{0} \sim$ $R / I_{0}$, d'après le lemme 2.11, $C$ est un intervalle de $R$ et $R^{\prime}$ sur lequel $R$ et $R^{\prime}$ sont réflexives ou irréflexives. Si $C$ est différente de sa composante connexe, alors le lemme 4.2 prouve que $R^{\prime} / C \sim R / C$. Dans la suite $C$ est égale à sa composante connexe. Comme $R^{\prime} / I_{0} \sim R / I_{0}, d=8$ et $k=4$, alors le lemme 2.13 prouve que $R / C$ et $R^{\prime} / C$ sont $(\leq 4)$-hypomorphes. Puisque $C$ n'est ni un tournoi sans diamant ni un élément de $E\left(S_{n}\right)$ alors d'après le lemme $2.7 R^{\prime} / C \sim R / C$. Il s'ensuit que $R^{\prime} \sim R$.

Lemme 6.7. Soient $R$ et $R^{\prime}$ deux relations binaires 8-hémimorphes sur une base finie $E$ de cardinal au moins égal $15, k$ le plus petit cardinal des restrictions non auto-duales de $R$, et $I_{0}$ la base d'une de ces restrictions.

Si $k=4$, si parmi les composantes connexes non auto-duales de $R$, une seule notée $D_{1}$ est un tournoi sans diamant ou un élément de $E\left(S_{n}\right)$, et si $R^{\prime} / I_{0} \sim R / I_{0}$, alors $R^{\prime} \sim R$ ou $R^{\prime} \sim R\left[D^{\star}{ }_{1}\right]$.

Preuve. Le lemme 2.5 prouve que les relations $R^{\prime}$ et $R$ sont $(\leq 8)$ hémimorphes. Comme $R^{\prime} / I_{0} \sim R / I_{0}$, alors d'après le lemme 2.11 toute classe de l'équivalence $D_{R, R^{\prime}}$ est un intervalle de $R$ et $R^{\prime}$ sur lequel $R$ et $R^{\prime}$ sont réflexives ou irréflexives. Le lemme 6.6 prouve que $R^{\prime} /\left(E-D_{1}\right) \sim$ $R /\left(E-D_{1}\right)$, d'autre part $R / D_{1}$ est connexe, alors le lemme 2.3 prouve que $R^{\prime} / D_{1} \sim R / D_{1}$ ou $R^{\prime} / D_{1} \sim R^{\star} / D_{1}$. Il s'ensuit que $R^{\prime} \sim R$ si $R^{\prime} / D_{1} \sim R / D_{1}$ et $R^{\prime} \sim R\left[D^{\star}{ }_{1}\right]$ si $R^{\prime} / D_{1} \sim R^{\star} / D_{1}$. 
Preuve du théorème 6.1. Soient $R$ et $R^{\prime}$ deux relations binaires 8hémimorphes sur une base finie $E$ de cardinal au moins égal à $15, k$ le plus petit cardinal des restrictions non auto-duales de $R$ et $I_{0}$ la base d'une de ces restrictions. Quitte à échanger les rôles de $R$ et $R^{\star}$, on va supposer que $R^{\prime} / I_{0} \sim R / I_{0}$. On a les cas suivants :

$C_{1}$. Si $R$ est connexe, alors le lemme 2.3 prouve que $R$ est 8 -demireconstructible.

$C_{2}$. Si $k=2$, alors le lemme 2.4 prouve que $R$ est 8 -demi-reconstructible.

$C_{3}$. Si $k=3$, on a :

$C_{31}$. Si $R$ admet au moins deux composantes connexes non auto-duales qui sont des tournois sans diamant et disjoints de tout drapeau, alors le lemme 6.2 prouve que $R$ n'est pas 8-demi-reconstructible.

$C_{32}$. Si chaque composante connexe de $R$ qui est un tournoi sans diamant est auto-dual où contient un sommet d'un drapeau, alors le lemme 6.3 prouve que $R$ est 8 -demi-reconstructible.

$C_{33}$. Si parmi les composantes connexes de $R$, une seule notée $D_{1}$ est un tournoi sans diamant non auto-dual disjoint de tout drapeau. Si $R^{\star}$ est isomorphe à $R\left[D^{\star}{ }_{1}\right]$, alors le lemme 6.4 prouve que $R$ est 8 -demireconstructible. Si $R^{\star}$ n'est pas isomorphe à $R\left[D^{\star}{ }_{1}\right]$, alors on peut voir que les relations $R$ et $R\left[D^{\star}{ }_{1}\right]$ sont $(\leq 8)$-hémimorphes sans être hémimorphes. Il s'ensuit que $R$ n'est pas 8-demi-reconstructible.

$C_{4}$. Si $k=4$, on a :

$C_{41}$. Si $R$ admet au moins deux composantes connexes non auto-duales qui sont des tournois sans diamant ou des éléments de $E\left(S_{n}\right)$, alors le lemme 5.2 prouve que $R$ n'est pas 8 -demi-reconstructible.

$C_{42}$. Si aucune composante connexe non auto-duale de $R$ n'est un tournoi sans diamant ou un élément de $E\left(S_{n}\right)$, alors le lemme 6.6 prouve que $R$ est 8-demi-reconstructible. 
$C_{43}$. Si parmi les composantes connexes non auto-duales de $R$, une seule notée $D_{1}$ est un tournoi sans diamant ou un élément de $E\left(S_{n}\right)$. Si $R^{\star}$ est isomorphe à $R\left[D^{\star}{ }_{1}\right]$, alors le lemme 6.7 prouve que $R$ est 8 -demireconstructible. Si $R^{\star}$ n'est pas isomorphe à $R\left[D^{\star}{ }_{1}\right]$, alors on peut voir que les relations $R$ et $R\left[D^{\star}{ }_{1}\right]$ sont ( $\left.\leq 8\right)$-hémimorphes sans être hémimorphes. Il s'ensuit que $\mathrm{R}$ n'est pas 8-demi-reconstructible.

$C_{5}$. Si $k=5$ et $R$ admet au moins deux composantes connexes non auto-duales qui sont des tournois sans diamant ou des éléments de $E\left(S_{n}\right)$, alors le lemme 5.2 prouve que $R$ n'est pas 8-demi-reconstructible. Si $R$ admet au plus une composante connexe non auto-duale qui est un tournoi sans diamant ou un élément de $E\left(S_{n}\right)$, alors le lemme 5.3 prouve que $R$ est 8-demi-reconstructible.

$C_{6}$. Si $k=6$ et $R$ admet au plus une composante connexe non autoduale qui est un tournoi sans diamant, alors le lemme 3.4 prouve que $R$ est 8-demi-reconstructible. Si $R$ admet au moins deux composantes connexes non auto-duales qui sont des tournois sans diamant, alors le lemme 3.5 prouve que $R$ n'est pas 8-demi-reconstructible.

$C_{7}$. Si $k \geq 7$ ou $k=0$. Dans ce cas $R$ n'admet aucune restriction non auto-duale de cardinal inférieur ou égal à 6 , alors le lemme 2.1 prouve que $R$ est 8-demi-reconstructible.

\section{Exemple de relation 9-demi-reconstructible mais non 8-demi- reconstructible.}

On considère :

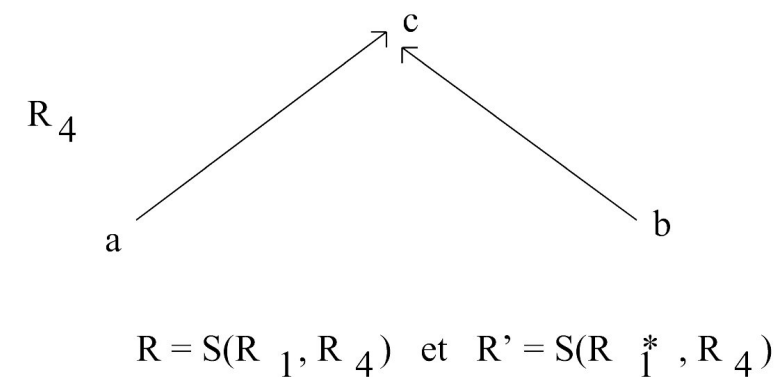


- La relation $R_{1}=C_{3}\left(T_{1}, T_{2}, T_{3}\right)$ où $C_{3}$ est un 3 -cycle de base $\{1,2,3\}$ et pour tout $i \in\{1,2,3\}, T_{i}$ est une $i$-chaîne irréflexive.

- La relation $R_{4}$ est une relation irréflexive à trois éléments $a, b$ et $c$ définie par : $R_{4}(a, b)=R_{4}(b, a)=-, R_{4}(a, c)=-R_{4}(c, a)=+$ et $R_{4}(b, c)=-R_{4}(c, b)=+$. $R_{4}$ s'appelle pic.

- $S$ : une relation binaire vide de base $\{1,2\}$.

Sous ces notations, on peut voir que les relations $R=S\left(R_{1}, R_{4}\right)$ et $R^{\prime}=S\left(R_{1}^{\star}, R_{4}\right)$ sont 8-hémimorphes, sans être hémimorphes, et par suite $R$ n'est pas 8-demi-reconstructible. Comme $R_{4}$ est un non auto-dual de cardinal 3, alors le lemme 2.4 prouve que $R$ est 9 -demi-reconstructible.

\section{Caractérisation des relations binaires 7-demi-reconstructibles}

Dans ce paragraphe nous obtenons :

Théorème 7.1. Soient $R$ une relation binaire de base finie $E$ de cardinal au moins égal 13 et $k$ le plus petit cardinal des restrictions non auto-duales de $R$. Alors $R$ n'est pas 7-demi-reconstructible si et seulement si $R$ vérifie l'une des conditions suivantes :

- $k=2$ et $R$ admet au moins deux composantes connexes non autoduales qui sont des tournois sans diamant.

- $k=2$, et parmi les composantes connexes de $R$, une seule notée $D_{1}$ est un tournoi sans diamant non auto-dual, et $R^{\star}$ n'est pas isomorphe à $R\left[D^{\star}{ }_{1}\right]$.

- $k=3, R$ admet au moins deux composantes connexes non auto-duales qui sont des tournois sans diamant ou des éléments de $E\left(S_{n}\right)$ disjoints de tout drapeau.

- $k=3$, et parmi les composantes connexes non auto-duales de $R$, une seule notée $D_{1}$ est un tournoi sans diamant ou un élément de $E\left(S_{n}\right)$ disjoint de tout drapeau, et $R^{\star}$ n'est pas isomorphe à $R\left[D^{\star}{ }_{1}\right]$. 
- $k=4$ et $R$ admet au moins deux composantes connexes non autoduales.

- $k=5$ et $R$ admet au moins deux composantes connexes non autoduales qui sont des tournois sans diamant ou des éléments de $E\left(S_{n}\right)$.

- $k=6$ et $R$ admet au moins deux composantes connexes non autoduales qui sont des tournois sans diamant.

La preuve du théorème 7.1 se base sur les résultats suivants :

Lemme 7.2. Soient $R$ et $R^{\prime}$ deux relations binaires 7-hémimorphes sur une base finie $E$ de cardinal au moins égal 13, $k$ le plus petit cardinal des restrictions non auto-duales de $R, I_{0}$ la base d'une de ces restrictions et $D$ une composante connexe de $R$.

Si $R / I_{0}$ est un drapeau, si $R^{\prime} / I_{0} \sim R / I_{0}$, si $R / D$ est un élément de $E\left(S_{n}\right)$ et si $D \cap I_{0} \neq \phi$ alors $R^{\prime} / D \sim R / D$.

Preuve. Le lemme 2.5 prouve que les relations $R^{\prime}$ et $R$ sont $(\leq 7)$ hémimorphes. Comme $R^{\prime} / I_{0} \sim R / I_{0}$, alors le lemme 2.11 prouve que toute classe de l'équivalence $D_{R, R^{\prime}}$ est un intervalle de $R$ et $R^{\prime}$ sur lequel $R$ et $R^{\prime}$ sont réflexives ou irréflexives. Si l'équivalence $D_{R / D, R^{\prime} / D}$ possède au moins deux classes, alors d'après le lemme $4.2 R^{\prime} / D \sim R / D$. Dans la suite $D$ est une classe de l'équivalence $D_{R, R^{\prime}}$. Comme $R / I_{0}$ est un drapeau, $d=7$, $R^{\prime} / I_{0} \sim R / I_{0}$, et $D \cap I_{0} \neq \phi$, alors le lemme 2.13 prouve que $R / D$ et $R^{\prime} / D$ sont ( $\leq 5$ )-hypomorphes. Puisque $R / D$ est un élément de $E\left(S_{n}\right)$, alors le lemme 3.2 prouve que $R^{\prime} / D \sim R / D$.

Lemme 7.3. Soient $R$ et $R^{\prime}$ deux relations binaires 7-hémimorphes sur une base finie $E$ de cardinal au moins égal 13, $k$ le plus petit cardinal des restrictions non auto-duales de $R, I_{0}$ la base d'une de ces restrictions et $D$ une composante connexe de $R$.

Si $k=3$, si $R$ n'admet aucune composante connexe qui est un tournoi sans diamant non auto-dual, si chaque composante connexe qui est un élément de $E\left(S_{n}\right)$ est auto-duale ou contient un sommet d'un drapeau, si de plus $R^{\prime} / I_{0} \sim R / I_{0}$ alors $R^{\prime} \sim R$.

Preuve. Le lemme 2.5 prouve que les relations $R^{\prime}$ et $R$ sont $(\leq 7)$ hémimorphes. Soit $C$ une classe de l'équivalence $D_{R, R^{\prime}}$. Comme $R^{\prime} / I_{0} \sim$ 
$R / I_{0}$, alors d'après le lemme $2.11 C$ est un intervalle de $R$ et $R^{\prime}$ sur lequel $R$ et $R^{\prime}$ sont réflexives ou irréflexives. Si $C$ est différente de sa composante connexe, alors le lemme 4.2 prouve que $R^{\prime} / C \sim R / C$. De même si $C$ est un élément de $E\left(S_{n}\right)$ et $C$ contient un sommet d'un drapeau, le lemme 7.2 prouve que $R^{\prime} / C \sim R / C$. Dans la suite $C$ est égale à sa composante connexe et $C$ n'est ni un tournoi sans diamant ni un élément de $E\left(S_{n}\right)$. Comme $R^{\prime} / I_{0} \sim R / I_{0}, d=7$ et $k=3$, alors le lemme 2.13 prouve que $R / C$ et $R^{\prime} / C$ sont $(\leq 4)$-hypomorphes, et par suite d'après le lemme 2.7 $R^{\prime} / C \sim R / C$. Il s'ensuit que $R^{\prime} \sim R$.

Lemme 7.4. Soient $R$ et $R^{\prime}$ deux relations binaires 7-hémimorphes sur une base finie $E$ de cardinal au moins égal $13, k$ le plus petit cardinal des restrictions non auto-duales de $R, I_{0}$ la base d'une de ces restrictions et $D$ une composante connexe de $R$.

Si $k=3$, si parmi les composantes connexes non auto-duales de $R$, une seule notée $D_{1}$ est un tournoi sans diamant ou un élément de $E\left(S_{n}\right)$ disjoint de tout drapeau, et si $R^{\prime} / I_{0} \sim R / I_{0}$, alors $R^{\prime} \sim R$ ou $R^{\prime} \sim R\left[D^{\star}{ }_{1}\right]$.

Preuve. Le lemme 2.5 prouve que les relations $R^{\prime}$ et $R$ sont $(\leq 7)$ hémimorphes. Comme $R^{\prime} / I_{0} \sim R / I_{0}$, alors d'après le lemme 2.11) toute classe de l'équivalence $D_{R, R^{\prime}}$ est un intervalle de $R$ et $R^{\prime}$, sur lequel $R$ et $R^{\prime}$ sont réflexives ou irréflexives. Le lemme 7.3 prouve que $R^{\prime} /(E-$ $\left.D_{1}\right) \sim R /\left(E-D_{1}\right)$, d'autre part $R / D_{1}$ est connexe, alors le lemme 2.3 prouve que $R^{\prime} / D_{1} \sim R / D_{1}$ ou $R^{\prime} / D_{1} \sim R^{\star} / D_{1}$. Il s'ensuit que $R^{\prime} \sim R$ si $R^{\prime} / D_{1} \sim R / D_{1}$ et $R^{\prime} \sim R\left[D^{\star}{ }_{1}\right]$ si $R^{\prime} / D_{1} \sim R^{\star} / D_{1}$.

Lemme 7.5. Soient $R$ une relation binaire sur une base finie $E$ et $k$ le plus petit cardinal des restrictions non auto-duales de $R$.

i) Si $R$ admet au moins deux composantes connexes $D_{1}$ et $D_{2}$ non auto-duales qui sont des tournois sans diamant, alors $R$ n'est pas 7-demireconstructible.

ii) Si $k=3$ et $R$ admet au moins deux composantes connexes $D_{1}$ et $D_{2}$ non auto-duales qui sont des tournois sans diamant ou des éléments de $E\left(S_{n}\right)$ disjoints de tout drapeau, alors $R$ n'est pas 7-demi-reconstructible.

iii) Si $k=4$ et $R$ admet au moins deux composantes connexes $D_{1}$ et $D_{2}$ non auto-duales, alors $R$ n'est pas 7-demi-reconstructible. 
Preuve. La preuve est analogue à celle du lemme 3.5, car dans la preuve du lemme 3.5 on n'a pas utilisé le fait que $D_{1}$ et $D_{2}$ sont des tournois sans diamant.

Preuve du théorème 7.1. Soient $R$ et $R^{\prime}$ deux relations binaires 7hémimorphes sur une base finie $E$ de cardinal au moins égal à $13, k$ le plus petit cardinal des restrictions non auto-duales de $R$ et $I_{0}$ la base d'une de ces restrictions. Quitte à échanger les rôles de $R$ et $R^{\star}$, on va supposer que $R^{\prime} / I_{0} \sim R / I_{0}$. On va distinguer les cas suivants :

$C_{1}$. Si $R$ est connexe, alors le lemme 2.3 prouve que $R$ est 7 -demireconstructible.

C2. Si $k=2$, on a :

$C_{21}$. Si $R$ admet au moins deux composantes connexes non auto-duales qui sont des tournois sans diamants, alors $i$ ) du lemme 7.5 prouve que $R$ n'est pas 7-demi-reconstructible.

$C_{22}$. Si $R$ n'admet aucune composante connexe non auto-duale qui est un tournoi sans diamant, comme $k=d-5(d=7$ et $k=2)$, alors le lemme 4.3 prouve que $R$ est 7 -demi-reconstructible.

$C_{23}$. Si parmi les composantes connexes non auto-duales de $R$, une seule notée $D_{1}$ est un tournoi sans diamant et si $R^{\star}$ est isomorphe à $R\left[D^{\star}{ }_{1}\right]$, alors le lemme 4.4 prouve que $R$ est 7 -demi-reconstructible. Si $R^{\star}$ n'est pas isomorphe à $R\left[D^{\star}{ }_{1}\right]$, alors on peut voir que les relations $R$ et $R\left[D^{\star}{ }_{1}\right]$ sont $(\leq 7)$-hémimorphes sans être hémimorphes. Il s'ensuit que $R$ n'est pas 7 demi-reconstructible.

$C_{3}$. Si $k=3$, on les sous cas suivants :

$C_{31}$. Si $R$ admet au moins deux composantes connexes non auto-duales qui sont des tournois sans diamants ou des éléments de $E\left(S_{n}\right)$, et si ces composantes connexes sont des éléments de $E\left(S_{n}\right)$ disjoints de tout drapeau, alors le $i i$ ) du lemme 7.5 prouve que $R$ n'est pas 7 -demi-reconstructible.

$C_{32}$. Si $R$ n'admet aucune composante connexe qui est un tournoi sans diamant non auto-dual, si chaque composante connexe qui est un élément 
de $E\left(S_{n}\right)$ est auto-duale ou contient un sommet d'un drapeau, alors le lemme 7.3 prouve que $R$ est 7 -demi-reconstructible.

$C_{33}$. Si parmi les composantes connexes non auto-duales de $R$, une seule notée $D_{1}$ est un tournoi sans diamant ou un élément de $E\left(S_{n}\right)$ disjoint de tout drapeau. Si $R^{\star}$ est isomorphe à $R\left[D^{\star}{ }_{1}\right]$, alors le lemme 7.4 prouve que $R$ est 7-demi-reconstructible. Si $R^{\star}$ n'est pas isomorphe à $R\left[D^{\star}{ }_{1}\right]$, alors on peut voir que les relations $R$ et $R\left[D^{\star}{ }_{1}\right]$ sont $(\leq 7)$-hémimorphes sans être hémimorphes. Il s'ensuit que $R$ n'est pas 7-demi-reconstructible.

$C_{4}$. Si $k=4$, et $R$ admet au moins deux composantes connexes non auto-duales, alors le $i i i)$ du lemme 7.5 prouve que $R$ n'est pas 7 -demireconstructible. Si $R$ admet au plus une composante connexe non autoduale, alors le lemme 2.3 prouve que $\mathrm{R}$ est 7 -demi-reconstructible.

$C_{5}$. Si $k=5$ et $R$ admet au moins deux composantes connexes non auto-duales qui sont des tournois sans diamant ou des éléments de $E\left(S_{n}\right)$, alors le lemme 5.2 prouve que $R$ n'est pas 7-demi-reconstructible. Si $R$ admet au plus une composante connexe non auto-duale qui est un tournoi sans diamant ou un élément de $E\left(S_{n}\right)$, alors le lemme 5.3 prouve que $R$ est 7-demi-reconstructible.

$C_{6}$. Si $k=6$ et $R$ admet au plus une composante connexe non autoduale qui est un tournoi sans diamant, alors le lemme 3.4 prouve que $R$ est 7 -demi-reconstructible. Si $R$ admet au moins deux composantes connexes non auto-duales qui sont des tournois sans diamant, alors le lemme 3.5 prouve que $R$ n'est pas 7-demi-reconstructible.

$C_{7}$. Si $k \geq 7$ ou $k=0$. Dans ce cas $R$ n'admet aucune restriction non auto-duale de cardinal inférieur ou égal à 6 , alors le lemme 2.1 prouve que $R$ est 7 -demi-reconstructible. 
Exemple de relation 8-demi-reconstructible mais non 7-demireconstructible

On considère :

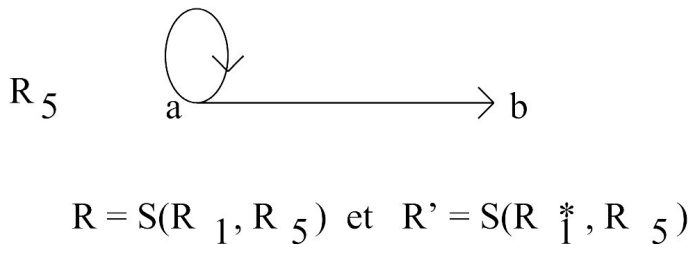

- La relation $R_{1}=C_{3}\left(T_{1}, T_{2}, T_{3}\right)$ où $C_{3}$ est un 3 -cycle de base $\{1,2,3\}$ et pour tout $i \in\{1,2,3\}, T_{i}$ est une $i$-chaîne irréflexive.

- La relation $R_{5}$ est une relation à deux éléments $a$ et $b$ définie par : $R_{5}(a, a)=-R_{5}(b, b)=+$ et $R_{5}(a, b)=-R_{5}(b, a)=+$.

- $S$ : une relation binaire vide de base $\{1,2\}$.

Sous ces notations, on peut voir que les relations $R=S\left(R_{1}, R_{5}\right)$ et $R^{\prime}=S\left(R_{1}^{\star}, R_{5}\right)$ sont 7-hémimorphes, sans être hémimorphes, et par suite $R$ n'est pas 7-demi-reconstructible. Comme $R_{5}$ est un non auto-dual de cardinal 2, alors le lemme 2.4 prouve que $R$ est 8 -demi-reconstructible.

\section{References}

[1] J. DAMMAK : La dualité dans la demi-reconstruction des relations binaires finies, CRAS, Série I 327 (1998), p. 861-864.

[2] R. FRAÏSSÉ : L'intervalle en théorie des relations, in orders, descriptions and roles, M. Pouzet et D. Richard éd. North-Holland, 1984, p. 313-342. 
[3] C. GNANVO et P.ILLE : La reconstruction des tournois. C. R. Acad. Sci. Paris, t. 306, série I, (1988), p. 577-580.

[4] J. G. HAGENDORF et G. LOPEZ : La demi-reconstructibilité des relations binaires d'au moins 13 éléments. C. R. Acad. Sci. Paris, série I, 317 (1993), P. 7-12.

[5] G. LOPEZ : L'indéformabilité des relations et multirelations binaires. Zeitschrift. Math. Logik Grundlagen Math. 24 (1978), p. 303-317.

[6] G. LOPEZ et C. RAUZY : Reconstruction of binary relations from their restrictions of cardinality 2,3,4 and (n-1), I. Zeitschr. f. Math. Logik und Grundlagen d. Math. Bd. 38, S. 27-37 (1992).

[7] G. LOPEZ et C. RAUZY : Reconstruction of binary relations from their restrictions of cardinality 2,3,4 and (n-1), II. Zeitschr. f. Math. Logik und Grundlagen d. Math. Bd 38, S. 157-168 (1992).

[8] M. POUZET : Application d'une propriété combinatoire des parties d'un ensemble aux groupes et aux relations. Math. Zeitschr. 150 (1976), p. 117-134.

Received : December 2002.

\section{J. Dammak}

Département de Mathématiques

Faculté des Sciences de Sfax

B.P. 802, 3018 Sfax

Tunisie

Fax 21674274437

e-mail : jdammak@yahoo.fr 
[3] C. GNANVO et P.ILLE : La reconstruction des tournois. C. R. Acad. Sci. Paris, t. 306, série I, (1988), p. 577-580.

[4] J. G. HAGENDORF et G. LOPEZ : La demi-reconstructibilité des relations binaires d'au moins 13 éléments. C. R. Acad. Sci. Paris, série I, 317 (1993), P. 7-12.

[5] G. LOPEZ : L'indéformabilité des relations et multirelations binaires. Zeitschrift. Math. Logik Grundlagen Math. 24 (1978), p. 303-317.

[6] G. LOPEZ et C. RAUZY : Reconstruction of binary relations from their restrictions of cardinality 2,3,4 and (n-1), I. Zeitschr. f. Math. Logik und Grundlagen d. Math. Bd. 38, S. 27-37 (1992).

[7] G. LOPEZ et C. RAUZY : Reconstruction of binary relations from their restrictions of cardinality 2,3,4 and (n-1), II. Zeitschr. f. Math. Logik und Grundlagen d. Math. Bd 38, S. 157-168 (1992).

[8] M. POUZET : Application d'une propriété combinatoire des parties d'un ensemble aux groupes et aux relations. Math. Zeitschr. 150 (1976), p. 117-134.

Received : December 2002.

\section{J. Dammak}

Département de Mathématiques

Faculté des Sciences de Sfax

B.P. 802, 3018 Sfax

Tunisie

Fax 21674274437

e-mail : jdammak@yahoo.fr 